\title{
Stability of Copper Nanoparticles in Media Imitating the Real Environment
}

\author{
Zuzana Bytešníková1, Martina Koláčková1, Pavel Švec¹, Anna Jánová1, Dalibor Húska1, Vojtěch Adam ${ }^{1,2}$, \\ Lukáš Richtera ${ }^{1,2}$, \\ ${ }^{1}$ Department of Chemistry and Biochemistry,Mendel University in Brno, \\ Zemědělská 1, 61300 Brno, Czech Republic \\ ${ }^{2}$ Central European Institute of Technology, Brno University of Technology, \\ Purkyňova 656/123, 61200 Brno, Czech Republic
}

zuzana.bytesnikova@mendelu.cz; martina.kolackova@mendelu.cz; xjanova3@node.mendelu.cz, pavel.svec@mendelu.cz dalibor.huska@mendelu.cz, vojtech.adam@mendelu.cz, lukas.richtera@mendelu.cz

\section{Extended Abstract}

In recent years, copper and copper-based nanoparticles (Cu NPs) have been used for industrial purposes. Cu NPs are increasingly used in various fields. Therefore, to understand the mechanism of copper toxicity to organisms, first it is essential to understand its dominance as a chemical and its behaviour in the environment. The fate of $\mathrm{Cu}$ NPs is nevertheless still unclear and also there is a lack of publications that study their stability to find out what really acts the nanomaterials or any other compound with copper in the different oxidative state. Copper is a naturally occurring ubiquitous element present in the environment however, at higher concentrations, $\mathrm{Cu}$ is generally toxic to plants and other organisms including fish, crustaceans, mussels, and algae. The fate and transformation of engineered $\mathrm{Cu}$ NPs in water environment must be intensively studied.

The synthesis of $\mathrm{Cu}$ NPs was inspired by Liu et al. [1] where the polyvinylpyrrolidone was replaced with sodium carboxymethylcellulose sodium salt (90kDa). The stability was studied using scanning electron microscopy (SEM) analysis, where back scattered electrons (BSE) were used for material contrast. Cu NPs were studied in tris-acetate-phosphate medium (TAP) to imitate the natural environment. As control was used deionized water. The concentrations of tested Cu NPs were $5,10,25,50$, and $100 \mathrm{mg} / \mathrm{L}$. The experiment was held for 96 hours in a normal light regime at laboratory temperature.

At the time of setting up an experiment, the material contrast of tested Cu NPs in TAP media as well as in deionized water was clearly observed. Already after 24 hours the contrast of the material was not observed using BSE and small flakes like object appear which is probably flakes of the polymer. The same trend was observed after the next 24 hours. Our results suggest that copper from $\mathrm{Cu}$ NPs was dissolved while the polymer disintegrated. The concentration dependence was observed. Dissolution of copper in NPs probably also changed the oxidation state of copper and therefore properties were also changed. The control $\mathrm{Cu}$ NPs stored in the same conditions in deionized water was not noticeable changed after the experiment. Moreover, BSE confirmed the presence of copper still in the core of $\mathrm{Cu}$ NPs.

The toxicological studies of nanomaterials should pay attention to their behaviour in the intended environment and nature of nanomaterials. This field still should be intensively studied forward to real environment testing to better understand the fate of nanomaterials and their toxicological effect.

Keywords: Nanomaterials, copper nanoparticles, environment, stability, pollution

\section{Acknowledgement:}

This work was financially supported by ERDF 'Multidisciplinary research to increase application potential of nanomaterials in agricultural practice' (No. CZ.02.1.01/0.0/0.0/16_025/0007314).

\section{References}

[1] Q. M. Liu, T. Yasunami, K. Kuruda, M. Okido, "Preparation of Cu nanoparticles with ascorbic acid by aqueous solution reduction method," Transactions of Nonferrous Metals Society of China, vol. 22, pp. 2198-2203, 2012. 